\title{
Novel experimental phenomena of fine-particle fluidized beds
}

\author{
Yuanfang $\mathrm{Fu}^{*}$, Dayou Liu \\ DES, Institute of Mechanics, Chinese Academy of Sciences, Beijing 100080, PR China
}

Received 5 December 2006; received in revised form 3 March 2007; accepted 4 March 2007

\begin{abstract}
The dynamic response of bed height and concentration waves in liquid-solid fluidized beds to a step change in the fluidization velocity is considered. We experimentally study the liquid-solid fluidized beds, spherical beadings, with sizes ranging from 230 to 270 mesh and the inner diameter of columns made from glass is $2.4 \mathrm{~mm}$. Experimental results find that under certain conditions, fine particles with large Richardson-Zaki exponent $n$ display different dynamic behavior from usual particles with smaller $n$ during expansion and collapse of the fluidized state.
\end{abstract}

(C) 2007 Elsevier Inc. All rights reserved.

Keywords: Two-phase flow; Bed height; Concentration shock wave; Fluidization

\section{Introduction}

Fluidized beds are common and important reactors in process engineering because of the good mass and heat transfer rate between the fluid and the particles, and between the particles and the sidewall. It is well known that there are non-uniform flow structures such as bubbles and slugs in fluidization [1]. Fluidization quality is closely related to the intrinsic properties of particles, e.g. particle density, particle size and size distribution, and also their surface characteristics. Following the rapidly growing application of fine-particle in industry, fluidization of fine particles has become a significant emerging filed [2].

In liquid-solid fluidized systems, when the particles possess zero net velocity $\left(u_{\mathrm{p}}=0\right)$, they are dispersed quite uniformly. When $U$ exceeds the $u_{\min }$. Various parameters in the fluidized bed, e.g., voidage $\alpha_{\mathrm{f}}$, fluid velocity $u_{\mathrm{f}}$, etc., can be determined according to the Richardson-Zaki relationship $U=u_{\mathrm{T}} \alpha_{\mathrm{f}}^{n}$ (Richardson and Zaki [3]). Slis et al. [4] extended the Richardson-Zaki relationship to the condition $u_{\mathrm{p}} \neq 0$. They used the relationship to develop a study

\footnotetext{
${ }^{*}$ Corresponding author. Tel.: +86 0162573765.

E-mail address: fuyf@imech.ac.cn (Y. Fu).
}

on the unsteady process in the fluidized bed. Slis et al. utilized to calculate the variation of bed height with time during the process of expansion or collapse under the smaller Richardson-Zaki $n$, and the result is well in agreement with experiment. Fan et al. [5], Van der Wielen [6] and Foscolo [7] are all made investigations into unsteady fluidization within particulate fluidized bed. Jin et al. [8], Jin [9], Liu and Jin [10] and Jiu and Lin [11] also deduced generalized Richardson-Zaki relationship and continuous wave equations from local equilibrium model (LEM). They use the relationship to develop study on unsteady process in the bed with arbitrarily changing velocity $U(t)$, and they also make research on the propagation and interaction of various kinds of concentration waves that under the smaller Richardson-Zaki $n$. They all use the smaller RichardsonZaki $n$ particles and obtain that during the expansion process of fluidization there only continuous concentration waves exist, and during the collapse process only concentration shock waves exist. In this paper, a systematic experiment is carried out in the process of fine-particle and large-particle during fluidization. The experimental results indicate that there exists entirely difference in wave characteristic and distribution of particle concentration under certain conditions during the fluidization process of large Richardson-Zaki $n$ and small Richardson-Zaki $n$ 
particles. The experiment shows when using small-particles (spherical beadings with diameter of $0.15-\mathrm{mm}$ ), there exist concentration shock waves not only during the collapse process but also expansion process. For example, during the expansion process, when the concentration of particle changes from 0.58 to 0.41 there exists a concentration shock wave moving up from the distributor, and at the time the shock waves arrive the bed surface the expansion process is in the end, and with the same concentration of particle there only exist continuous concentration waves during the collapse process. However when the concentration of particle change from 0.37 to 0.17 there exists a series of continuous concentration waves moving up from the distributor during the expansion process, and there only exists a concentration shock wave during the collapse process, which are identical to the results of the researchers before.

For larger particles ( small $n$ ), shock wave only exists in the collapse process and during the expansion process only continuous waves occur, it is a hackneyed phenomenon. But for smaller particles (with large $n$ ), under certain conditions, during collapse process only exist continuous waves and during expansion process the shock wave occurs, it is a new phenomenon that beyond the earlier research works, it is not opened out by the earlier researchers. The works on this new phenomenon can help researchers study the fluidized beds deeply, and is meaningful for basic research.

\section{Experimental facility}

Fig. 1 is the schematic diagram of the experimental facility. The fluidization experiments are carried out in a 2.4$\mathrm{mm}$ inner diameter and 1.2- $\mathrm{m}$ high glass column, the fluidizing liquid is introduced at the bottom of the column through a calming section packed with small glass beads of $0.012-\mathrm{m}$ length and a sieve plate, from which relatively high resistance to flow to insures the two-phase flow in

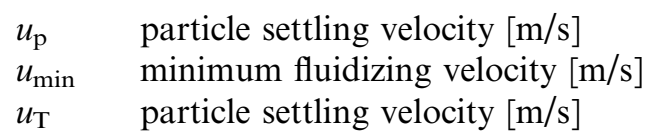

Greek letters

$\alpha_{\mathrm{f}} \quad$ voidage (fluid volume fraction)

$\alpha_{\mathrm{f} 1} \quad$ fluid volume fraction correspond to $U_{1}$

$\alpha_{\mathrm{f} 2} \quad$ fluid volume fraction correspond to $U_{2}$

$\alpha_{\mathrm{p}, \mathrm{c}} \quad$ packed particle volume fraction

$\rho_{\mathrm{f}} \quad$ fluid density $\left[\mathrm{kg} / \mathrm{m}^{3}\right]$

$\rho_{\mathrm{p}} \quad$ material density of particle $\left[\mathrm{kg} / \mathrm{m}^{3}\right]$

the bed to be essentially one-dimensional. In all experiments, cool boiled water was used as the fluidizing liquid, water from the compressed water reservoir (5) flows down to fluidize the glass beads in fluidized bed (13), the flow rate is measured by means of a flow meter or an indirect method. The bed height and the transient distribution of the particle concentration are recorded by the digital camera or digital vidicon. Properties of the experimental particles and fluid and experiment condition are listed in Table 1 . The beads are sieved carefully between two close

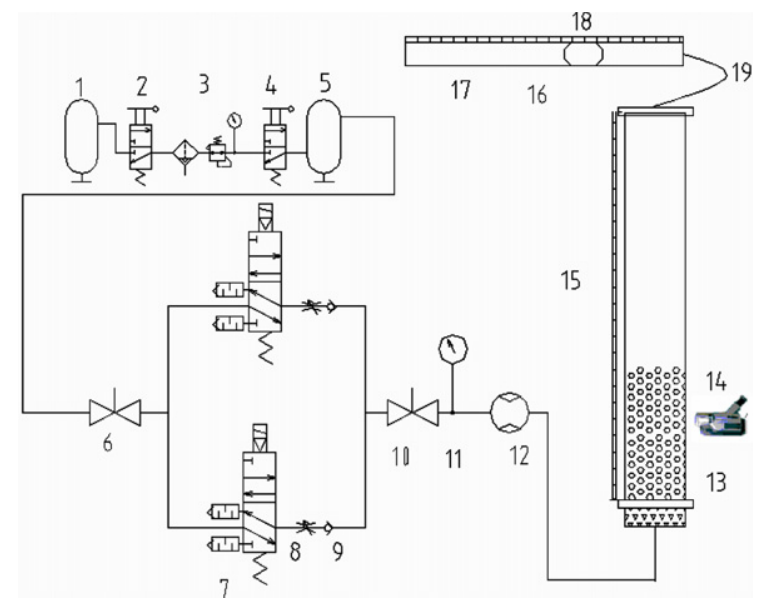

Fig. 1. Schematic diagram of experimental facility of fine-particle fluidized beds. (1) Nitrogen cylinder, (2) start valve, (3) filter regulator, (4) start valve, (5) compressed water reservoir, (6) cut-off valve, (7) solenoid valve, (8) precision flow control valve, (9) non-return valve, (10) cut-off valve, (11) pressure gauge, (12) flowmeter, (13) fluidized bed, (14) DV/DC, (15) ruler, (16) air bubble, (17) glass pipe, (18) ruler, (19) tube.

Table 1

Properties of particle, fluid and experiment condition

\begin{tabular}{llllllll}
\hline $\begin{array}{l}\rho_{\mathrm{p}} \\
\left(\mathrm{kg} / \mathrm{m}^{3}\right)\end{array}$ & $\begin{array}{l}d_{\mathrm{p}} \\
(\mathrm{m})\end{array}$ & $\begin{array}{l}\rho_{\mathrm{f}} \\
\left(\mathrm{kg} / \mathrm{m}^{3}\right)\end{array}$ & $\begin{array}{l}h_{0} \\
(\mathrm{~m})\end{array}$ & $\begin{array}{l}u_{\mathrm{T}} \\
(\mathrm{m} / \mathrm{s})\end{array}$ & $\begin{array}{l}u_{\min } \\
(\mathrm{m} / \mathrm{s})\end{array}$ & $n$ & $\alpha_{\mathrm{pc}}$ \\
\hline $2.6 \mathrm{E} 3$ & $1.5 \mathrm{E}-4$ & $1 \mathrm{E} 3$ & 0.25 & $2.67 \mathrm{E}-2$ & $6.3 \mathrm{E}-5$ & 4 & 0.6 \\
\hline
\end{tabular}




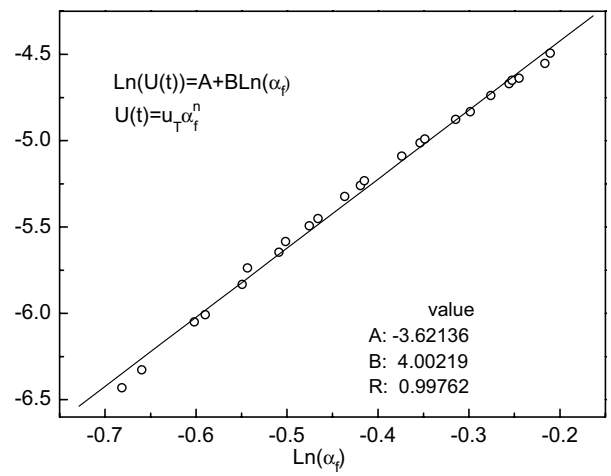

Fig. 2. Richardson-Zaki ln-ln plot.

meshes. The particle density $\rho_{\mathrm{p}}$ is determined by measuring the volume variation when a known mass of particles was introduced into a known volume of water in a graduated vessel. To determine the Richardson-Zaki exponent $n$ and particle terminal velocity, a number of steady-state experiments were conducted to determine the relationship between bed height and fluidization velocity. A linear regression of the $\log -\log$ plot resulted in a RichardsonZaki exponent is 4.00 and particle settling velocity is $0.0267 \mathrm{~m} / \mathrm{s}$, with a correlation coefficient of 0.9976 (see Fig. 2).

\section{Experimental methods}

Design of steady small flux liquid feed system is indicated as follows: to meet with the small flux by fine particles and fine tube fluidization experiment, we set up a set of small flux liquid feed system, which is proved by high-pressure nitrogen compressing liquid storage. No.1 in Fig. 1 is nitrogen cylinder with the pressure of $15 \pm$ $0.5 \mathrm{MPa}$ and capacity of $6-\mathrm{m}^{3}$. High-pressure nitrogen in cylinder presses the liquid surface of liquid storage through a series of adjustment and filtration, and the pressure on the liquid surface can be adjusted according to the experiment. Liquid storage No. 5 is loaded with cool boiled water, since it can avoid overflow of the bubble in liquid when to be affected by temperature and pressure change and do good to experiment result. Liquid from storage in given pressure flows into fluidized bed through ball valve, magnetic valve, precise flow control valve, check valve, pressure meter and flow meter. Fluidization velocity can be changed by adjusting precise flow control valve and opening different magnetic valves, and thus, different flow specified by dynamic course of bed layer can be acquired. Besides, to eliminate the influence of bubbles attached on particles, particles need to be soaked in cool boiled water and stirred continuously, and then be sucked by vacuum pump after $24 \mathrm{~h}$ soak. The bed height was recorded by a digital vidicon as function of time. A ruler was placed axially along the column in order to determine the bed height. This method has an accuracy of approximately $\pm 1-\mathrm{mm}$. The solid-liquid interface remained sharp for the step changes performed throughout all the experimental parameters. Since the particle is very fine and the flow needed is very small, how to truly response to the change of fluidization velocity will have great impact on experimental result. To measure change of velocity correctly, a section of tube (Fig. 1 (19)) is used to make a connection between the outlet of fluidized bed and one section of horizontal glass pipe (Fig. 1 (17)) that has the same diameter with the column of the bed. Air bubble (Fig. 1 (16)) is injected by a needle tube from the inlet of the horizontal glass pipe. Therefore, the movement of the bubble can be recorded by the digital vidicon clearly, via appropriate data processing; we can get the curves which is the reflection of the actual flow velocity changing process. The results show that the change of fluidization velocity is very close to stepwise.

\section{Experimental results and discussion}

In all experiments, the bed is in stead-state operating conditions before a stepwise change of fluidization velocity $U$ was introduced. Under steady-state condition, the voidage for a particulate fluidized bed is uniform throughout the bed. The bed experience a step increase of fluidization velocity from $U_{1}$ to $U_{2}$ for expansion experiments and a stepwise decrease from $U_{2}$ to $U_{1}$ for collapse experiments where $U_{2}>U_{1}$ always holds up.

The experiment results show that when the particles are small enough, that is, the Richardson-Zaki $n$ is large enough, there only exist concentration shock waves (see Fig. 3a) under some given conditions during the collapse process, the shock waves arrive the bed surface and at the time the collapse process is finishes (see Fig. 4a), which is theoretically and experimentally in agreement with the results of the researchers before. In addition, another important result is found by the experiment: when to use fine particles, it also appears concentration shock waves during the process of expansion (see Fig. 3b) under certain conditions, and the waves move upward from the distributor, the whole expansion process is completed as soon as the shock waves arrive bed surface (see Fig. 4b).
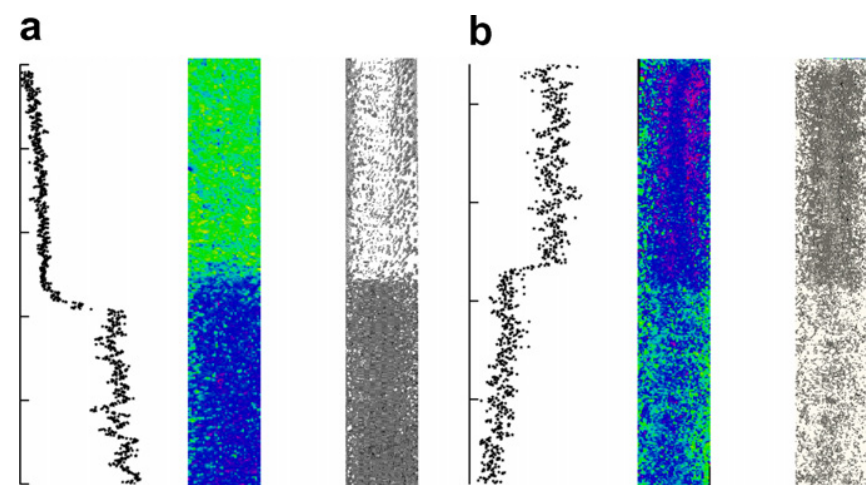

Fig. 3. Transient distribution of concentration shock wave in beds during the collapse and expansion process. (a) Concentration changes from 0.17 to 0.37 and (b) concentration changes from 0.58 to 0.41 . 

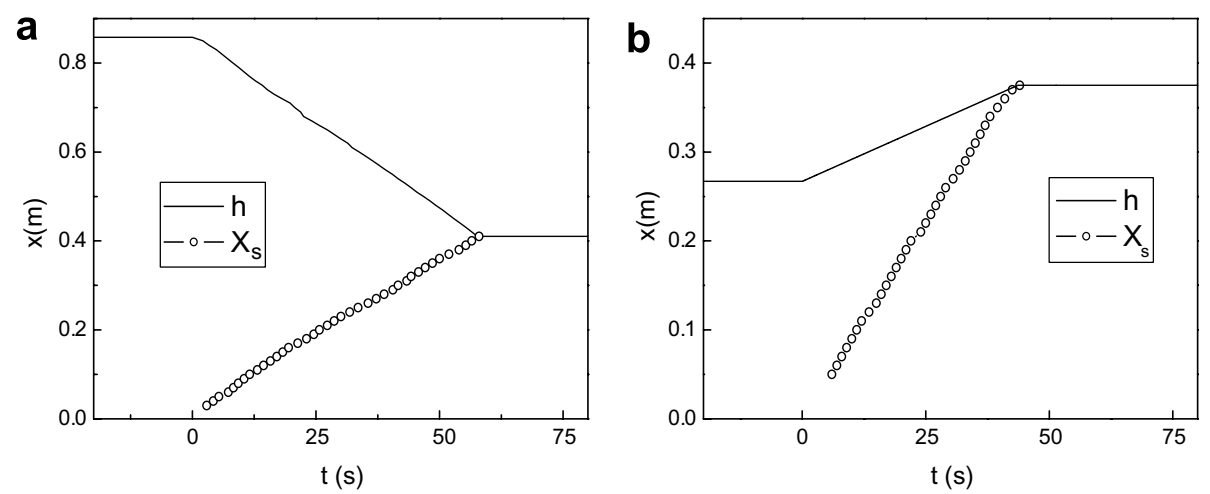

Fig. 4. Trace of bed height and concentration shock wave resulting from a sudden increase or decrease of fluidizing velocity.

\section{Acknowledgements}

This work was supported by the National Natural Science Foundation of China (NSFC, No. 20376083) and the Multiphase Reaction Laboratory, Institute of Process Engineering, Chinese Academy of Sciences.

\section{References}

[1] D.J. Needham, J.H. Merkin, The propagation of a voidage disturbance in a uniformly fluidized bed, J. Fluid Mech. 13 (1983) 427-454.

[2] M. Kwauk, Fluidization, Science Press, Beijing, 1992.

[3] J.F. Richardson, W.N. Zaki, Sedimentation and fluidization: part I, Trans. Inst. Chem. Engrs. 32 (1954) 35-53.

[4] P.L. Slis, TH.W. Willemse, H. Kramers, The response of the level of a liquid fluidized bed to a sudden change in the fluidizing velocity, Appl. Sci. Res. A 8 (1959) 209-218.
[5] L.T. Fan, J.A. Schmitz, E.N. Miller, Dynamics of liquid-solid fluidized bed expansion, AIChE J. 9 (2) (1963) 149-153.

[6] L.A.M. Van der Wielen, A.W.K.G. Sjauw Koen Fa, J.J.M. Potters, K.Ch.A.M. Luyben, Transient behavior of pulsed particulate fluidized beds, AIChE J. 43 (3) (1997) 625-630.

[7] P.U. Foscolo, L.G. Gibilaro, S.P. Waldram, A unified model for particulate expansion of fluidized beds and flow in fixed porous media, Chem. Eng. Sci. 38 (8) (1983) P1251-P1260.

[8] G.D. Jin, Y.S. Nie, D.Y. Liu, Numerical simulation of pulsed liquid fluidization bed and its experimental validation, Powder Technol. 119 (2001) 153-163.

[9] G.D. Jin, Numerical Simulation of Liquid Pulsed Fluidization and its Experimental Validation. Ph.D. Dissertation, Institute of Mechanics, Chinese Academy of Sciences, Beijing, 2002, (in Chinese).

[10] D.Y. Liu, G.D. Jin, Modeling two-phase flow in pulsed fluidized bed, China Particuology 1 (3) (2003) 95-104.

[11] G.D. Jin, D.Y. Liu, Modeling and simulation of liquid pulsed particulate fluidized beds, Powder Technol. 154 (2005) 138-155. 